\title{
A importância do profissional da nutrição na orientação adequada dos praticantes do
}

\section{vegetarianismo}

\author{
The importance of the nutrition professional in guiding vegetarian practitioners \\ La importancia del profesional de la nutrición para guiar a los practicantes vegetarianos
}

Recebido: 27/09/2021 | Revisado: 04/10/2021 | Aceito: 05/10/2021 | Publicado: 09/10/2021

\author{
Bruna Karine Bastos de Vasconcelos \\ ORCID: https://orcid.org/0000-0003-4503-9817 \\ Centro Universitário Fametro, Brasil \\ E-mail: bkvasconcelos5@gmail.com \\ Monique dos Santos Farias \\ ORCID: https://orcid.org/0000-0002-6408-6304 \\ Centro Universitário Fametro, Brasil \\ E-mail: santosmonique856@gmail.com \\ Daize Milena Costa da Silva \\ ORCID: https://orcid.org/0000-0003-0335-1845 \\ Centro Universitário Fametro, Brasil \\ E-mail: dayzemilena@gmail.com \\ Francisca Marta Nascimento de Oliveira Freitas \\ ORCID: https://orcid.org/0000-0002-0044-0925 \\ Centro Universitário Fametro, Brasil \\ E-mail: Francisca.freitas@fametro.edu.br
}

\begin{abstract}
Resumo
Introdução: No ramo da alimentação vegetariana pode-se identificar várias diferenças dessa prática. $\mathrm{O}$ termo vegetarianismo em si remete a uma dieta isenta de carne animal. Objetivo Geral: O presente artigo é parte de uma pesquisa que tem como objetivo mostrar os diferentes aspectos da prática do veganismo e vegetarianismo, seu impacto na saúde, e a influência do nutricionista nessa prática. Assim como descrever os nutrientes na composição alimentar e recomendação na dieta vegetariana. Metodologia: $\mathrm{O}$ trabalho foi desenvolvido com coleta de dados a parti de fontes segundarias, por meio de levantamento bibliográfico e baseado no estudo de autores e suas pesquisas sobre o vegetarianismo e o impacto na saúde humana. Resultado e discussão: Para realização desse estudo foi desenvolvido com base nos artigos o impacto deste tipo de dieta na vida adulta. Estas pesquisas foram realizadas em base de artigos científicos, publicações acadêmicas e periódicos científicos, em maioria dos últimos 10 anos. Também foram utilizados livros acadêmicos e científicos como material de consulta. Conclusão: Conclui-se com realização desse projeto orientar os indivíduos vegetarianos sobre a importância do nutricionista ao iniciar esse tipo de alimentação, pontuando os riscos da falta de informação que pode causar inúmeras desordens alimentares, como as deficiências nutricionais que podem ser supridas tanto pela dieta, adequando os nutrientes e respeitando as recomendações quanto, e se preciso, fazer o uso da suplementação.
\end{abstract}

Palavras-chave: Filosofia alimentar; Veganismo; Vegetarianismo; Nutricionista.

\begin{abstract}
Introduction: In the field of vegetarian food, several differences in this practice can be identified. The term vegetarianism itself refers to a diet free from animal meat. General Objective: This article is part of a research that aims to show the different aspects of the practice of veganism and vegetarianism, their impact on health, and the influence of nutritionists in this practice. As well as describing the nutrients in the food composition and recommendation in the vegetarian diet. Methodology: The work was developed with data collection from secondary sources, through a bibliographic survey and based on the study of authors and their research on vegetarianism and its impact on human health. Results and discussion: To carry out this study, the impact of this type of diet in adult life was developed based on the articles. These researches were carried out on the basis of scientific articles, academic publications and scientific journals, in most of the last 10 years. Academic and scientific books were also used as reference material. Conclusion: We conclude with this project to guide vegetarian individuals about the importance of a nutritionist when starting this type of diet, pointing out the risks of lack of information that can cause numerous eating disorders, such as nutritional deficiencies that can be supplied by both the diet, adapting the nutrients and respecting the recommendations when, and if necessary, to use the supplementation.
\end{abstract}

Keywords: Food philosophy; Veganism; Vegetarianism; Nutritionist. 


\begin{abstract}
Resumen
Introducción: En el campo de la comida vegetariana, se pueden identificar varias diferencias en esta práctica. El término vegetarianismo en sí se refiere a una dieta libre de carne animal. Objetivo general: Este artículo es parte de una investigación que tiene como objetivo mostrar los diferentes aspectos de la práctica del veganismo y el vegetarianismo, su impacto en la salud y la influencia de los nutricionistas en esta práctica. Además de describir los nutrientes en la composición de los alimentos y recomendaciones en la dieta vegetariana. Metodología: El trabajo se desarrolló con la recolección de datos de fuentes secundarias, a través de una encuesta bibliográfica y con base en el estudio de los autores y sus investigaciones sobre el vegetarianismo y su impacto en la salud humana. Resultados y discusión: Para la realización de este estudio se desarrolló el impacto de este tipo de dieta en la vida adulta a partir de los artículos. Estas investigaciones se han realizado sobre la base de artículos científicos, publicaciones académicas y revistas científicas, en la mayor parte de los últimos 10 años. También se utilizaron libros académicos y científicos como material de referencia. Conclusión: Concluimos con este proyecto para orientar a las personas vegetarianas sobre la importancia de un nutricionista a la hora de iniciar este tipo de dietas, señalando los riesgos de falta de información que pueden ocasionar numerosos trastornos alimentarios, como las deficiencias nutricionales que pueden ser suplidas tanto por la dieta., adaptando los nutrientes y respetando las recomendaciones cuando, y si es necesario, utilizar la suplementación.
\end{abstract}

Palabras clave: Filosofia alimentaria; Veganismo; Vegetarianismo; Nutricionista.

\title{
1. Introdução
}

A busca pela filosofia alimentar vegetariana muda crescentemente ao decorrer das décadas, por diferentes causas e modos da realização dessa pratica, já que, visualiza-se os alimentos de origem vegetal como possibilidade de uma melhor qualidade de vida e que podem assim serem responsáveis a uma maior longevidade (Slywitch, [20--], p. 02).

Esse tipo de alimentação tem classificações que incluem veganos, semivegetarianos, lactovegetarianos, ovovegetarianos, ovolactovegetarianos. Cada tipo exclui ou inclui certos alimentos (Gonzaga, 2014).

A União Vegetariana Internacional (UVI) diz que os vegetarianos não ingerem nenhum tipo de carne (boi, suínos, aves, peixe, frutos do mar, etc.), embora alguns consumam alguns produtos proveniente dos animais, tais como ovos, leite e seus derivados. A restrição dos produtos provenientes dos animais é o que determina o tipo de vegetarianismo, que pode variar desde os ovo-lacto-vegetarianos que consomem leites, ovos e derivados, até os veganos (vegans ou vegetarianos puros) que não consomem nenhum produto animal (Nascimento \& Sawyer, 2007).

Franco e Rego (2005) e Sant'ana (2008) distinguem esse grupo em cinco estratos. O ovo-lacto-vegetariano que não come carne, masconsomeovos, mel e leite, logo seus derivados; o lacto-vegetariano que não consomem carne, nem ovos, mas consomem laticínios e podem consumir mel; o ovo-vegetariano que não consomem carne e nem laticínios, mas consome ovos e pode consumir mel; o frugívoro ou frutívoro que além da recusa em contribuir para exploração e morte do animal, também se recusa a participar da morte das plantas, recusa usar o couro e produtos testados em animais (dependendo do grau desta dieta, o grupo poderá enquadrar-se no veganismo). E, por fim, o vegano que exclui de sua dieta todos os tipos de alimentos que contenha origem animal, desde a carne até o mel, ovo, leite entre muitos outros. O vegetariano também adota outra postura que o diferencia bastante dos outros grupos, pois além dos alimentos, eles também não utilizam outros artigos que advenham de origem animal, como o couro (cinto, sapato, jaqueta), cosméticos que são testados em animais, entre tantos outros.

A dieta em questão por ser de cunho restritivo acaba gerando grande debate social. Pela popularização dessa filosofia se questiona a prevenção de deficiências que abrange a promoção da saúde como prioridade, mas, considerando fatores socioeconômicos, culturais, destruição ambiental, sofrimento animal, na conveniência pessoal ou facilidade de elaboração dessa dieta (Baena, 2015).

Segundo o Guia Alimentar de Dietas Vegetarianas (2012), a orientação nutricional vinda de um profissional da nutrição é a base da dieta de um vegetariano que supra as determinadas necessidades nutricionais dos praticantes, visando o conforto e a melhor combinação possível para o desenvolvimento dessa escolha alimentar, pontuando os riscos, que se dão 
pela prática, sem as reposições necessárias dos nutrientes, e os benefícios que causam a saúde como prevenir e tratar as doenças crônicas.

Pesquisas realizadas com base nessa alimentação, mostram benefícios quanto às doenças, especialmente as crônicas. Há também pesquisas que mostram aspectos negativos, provocando deficiências nutricionais quando a dieta é restritiva ou desequilibrada. As dietas vegetarianas, quando bem planejadas, promovem o desenvolvimento adequados e podem ser adotadas em todos os ciclos da vida, inclusive por atletas, na gestação, infância e terceira idade. Quando se censura a ingestão de um determinado alimento dentro de uma dieta, haverá a necessidade de substituição por outro alimento que fornecerá os mesmos nutrientes ou nutrientes semelhantes para com a necessidade nutricional do indivíduo. Por isso a importância da atuação profissional de nutrição para orientação correta no estilo de vida. (Slywitch, 2012)

Os objetivos da pesquisa são mostrar os diferentes aspectos da prática do veganismo e vegetarianismo, seu impacto na saúde, e a influência do nutricionista nessa pratica. Assim como, descrever os diferentes aspectos da prática do veganismo e vegetarianismo. Os nutrientes na composição alimentar e recomendação no vegetarianismo. A importância do profissional de nutrição no acompanhamento desta filosofia alimentar.

\section{Metodologia}

Para a realização desse estudo optou-se por uma pesquisa na modalidade de revisão descritiva da literatura. A revisão descritiva propõe a identificação, registro e análise das características, fatores ou variáveis que se relacionam com o fenômeno ou processo (Perovano, 2014). Dessa forma, embasada em referências bibliográficas e científicas, relatando o merecimento do profissional na nutrição na orientação adequada dos praticantes do vegetarianismo.

O trabalho foi desenvolvido com coleta de dados a partir de fontes secundárias, mediante de levantamento bibliográfico e baseado no estudo de autores e suas pesquisas sobre o vegetarianismo e o impacto na saúde humana.

Onde foi estudado com base nos artigos o impacto desta dieta na vida adulta. Estas pesquisas foram realizadas em base dentre artigos científicos, estudos acadêmicos, e periódicos científicos entre 5 e 10 anos. Além disso foram aproveitados livros acadêmicos e científico como ferramenta de consulta. Buscou-se através das plataformas as pesquisas com os seguintes descritores, no idioma português: " biodisponibilidade ", "alimentação vegetariana ", " vegetarianismo “, " vegetarianos “, " dieta vegetariana ", " nutrientes essenciais ".

Através dessas pesquisas, resultaram 50 artigos em qualquer base de dados, tornando-se excluso 10 divulgações acadêmicas que não respondiam a questão norteadora desse estudo, levando assim, a inclusão de 40 divulgações que atenderam o modo de inclusão.

As coletas das divulgações foram examinadas em quatro fase. Nas primeiras duas fases, foram realizadas as buscas dos descritores que norteariam esse estudo, excluindo aqueles que não apresentam relação com o que este estudo propõe. Nas duas últimas fases analisou-se detalhadamente os artigos restantes, tendo como objetivo a proposta de incluir apenas artigos que tenham relação direta com o tema e que contemplem o tempo preestabelecido.

Foram escolhidos os estudos que abordam a temática com clareza, detalhando o conteúdo proposto e que respondam a questão norteadora proposto por este presente trabalho.

\section{Resultados e Discussão}

\subsection{Diferentes aspectos da prática do veganismo e vegetarianismo}

No ramo da alimentação denominada vegetariana identifica-se várias diferenças dessa pratica. O termo vegetarianismo em si remete a uma dieta isenta de carne animal. Por possuírem a mesma razão o veganismo faz parte dessa mesma filosofia, mas ele é o vegetarianismo restrito onde exclui todos os alimentos que são provenientes dos animais (carnes, 
peixes, leite, ovos e, às vezes, mel) e tem como fonte de alimento os produtos como cereais, leguminosas, frutas, verduras e oleaginosas (Azevedo, 2013).

Essa restrição alimentar tem como motivação a ética, porém existem praticantes que optam por ela em razão da saúde. Uma diferença notória uma vez que entre os vegetarianos é que possuem motivações diferentes dos veganos. Além de ser considerado um estilo de vida, os veganos mudam todo o cotidiano, pois excluem produtos que vem dos animais e não só os alimentos, como os vegetarianos (Dias et al., [entre 2017 e 2021]). As dietas vegetarianas, quando organizadas, proporcionam macro e micronutrientes em quantidades adequadas, além de inúmeras substâncias ativas, como fitoquímicos e fibras, que adapta benefícios a saúde. Da mesma forma, contribuem para diminuir os gastos de energia e de substâncias associadas ao crescimento de enfermidades crônicas (Baena, 2015).

A EPIC OXFORD (coorte de Oxford sobre Investigação Prospectiva Europeia de Câncer e Nutrição) efetuou um estudo transversal de análise alimentar que confronta o IMC de quatro grupos com dietas diferentes (consumidores de carne, peixe, vegetarianos e veganos). Foi considerado que os consumidores de peixe, os vegetarianos e principalmente os veganos mostram o menor índice de massa corporal (IMC) quando se comparam aos consumidores de carne. Entre as possíveis explicações para esta associação estão o grande consumo de fibras, grãos integrais, nozes e sementes, como a menor ingestão de gorduras e energias em geral pelos vegetarianos (Spencer et al., 2003).

Do mesmo modo, o Adventist Health Study-2 (AHS-2), uma coorte norte-americana, estudou a relação dos padrões alimentares vegetarianos com o risco a saúde e de crescimento de doenças. Os autores comprovaram que padrões alimentares vegetarianos foram associados à índices menores de: IMC; prevalência e incidência de diabetes mellitus; prevalência da síndrome metabólica e seus componentes; prevalência de hipertensão e entre outros, e, pode acontecer em alguns casos, menor risco de desenvolvimento de câncer. O resultado inicial demonstra ligações essenciais entre padrões alimentares vegetarianos e melhoria da saúde (Orlich \& Fraser, 2014).

\subsection{Os nutrientes na composição alimentar e recomendação na dieta vegetariana}

O corpo humano não tem necessidade de carne ou leite animal e não há nenhum nutriente indispensável para uma boa saúde nesses alimentos, que não se possa obter nas plantas (RODRIGUES, 2005). Entre as características que cercam o consumo da alimentação vegetariana, nota-se um maior consumo de fibras solúveis e não solúveis, vitamina $\mathrm{C}$, e de outros alimentos que contribuem de forma fitoquímica (Craig \& Mangels, 2009), consumindo também em maior quantidade vegetais e hortaliças e uma menor quantidade de gorduras saturadas (Couceiro, Lenz \& Slywitch, 2008). Sendo assim, nota-se que uma quantidade diminuta das pessoas que aderem o estilo de vida vegetariano tem uma baixa ingesta de alimentos que tem em sua composição a Vitamina B12, Cálcio, Zinco, o ômega-3, e demais micro e macro nutrientes (Craig \& Mangels, 2009).

\subsection{1 Ômega 3}

É um tipo de ácido graxo poliinsaturado, substância muito importante para o organismo, mais comumente encontrada em alimentos marinhos, pode prevenir doenças cardiovasculares, metabolismo de triglicerídeos, plaquetas e função endotelial (Gomes et al., 2014).

No corpo humano, quando o ômega 3 é consumido, ele é convertido em suas formas ativas EPA (ácido eicosapentaenóico) e DHA (ácido docosahexaenóico). Portanto, a DRI recomenda ômega 3 para vegetarianos. Deve ser mais do que onívoros 3,2 gramas para homens com mais de 14 anos e 2,2 gramas para mulheres maiores de 14 anos (Slywitch, 2012). Nozes, óleos e sementes de linhaça, soja, sementes de chia, beldroegas e outros alimentos (Slywitch, 2012). Nozes, óleo e sementes de linhaça, soja, sementes de chia, portulaca e algas marinhas, sejam tomados por via oral ou suplementados em 
cápsulas, podem ajudar a equilibrar o equilíbrio do ômega-3. Apesar da baixa ingestão, não há evidências de que os vegetarianos carecem desse nutriente (Borges et al., 2015; Marques et al.,2016).

\subsubsection{Zinco}

Na dieta vegetariana, o zinco precisa de atenção especial na sua ingestão, pois tem uma absorção moderada, e é considerado uma boa fonte de zinco por não comer carne (Marques et al.,2016).

Segundo a DRISS, a prescrição dietética recomendada para este mineral é 50\% superior à prescrição para onívoros, que é de 16,5 mg para homens com mais de 14 anos e $12 \mathrm{mg}$ para mulheres com mais de 19 anos (Slywitch, 2012).

De acordo com as diretrizes dietéticas para adultos vegetarianos, os alimentos ricos em vitamina $\mathrm{C}$ podem estimular o zinco porque ele pode ser inibido pelo ácido fítico. Para atingir a suficiência de zinco no corpo, deve-se comer alimentos ricos em vitamina C, pão integral, grãos, feijão e sementes. Embora menos do que onívoros, a ingestão de zinco em uma dieta vegetariana é considerada suficiente (Castillo \& Ortigosa, 2017; Slywitch, 2012; Borges et al., 2015).

\subsubsection{Iodo}

O iodo é uma parte importante da função da tireóide e ajuda o crescimento e a função do cérebro e do sistema nervoso central. Essa baixa ingestão de micronutrientes pode causar problemas como bócio, hipotireoidismo e retardo mental em qualquer fase da vida (Nadal e Mezzomo, 2016). Para os vegetarianos, não ingerir alimentos fortificados e suplementados, como sal iodado e algas marinhas, pode levar a um baixo teor de iodo no corpo (Borges et al., 2015), enquanto os alimentos vegetais têm baixa biodisponibilidade de iodo (Cozzolino, 2016)). De acordo com o DRI, a ingestão diária de iodo recomendada para adultos vegetarianos e onívoros adultos é de 100 a $150 \mathrm{mg} /$ dia (Cozzolino, 2016).

\subsubsection{Vitamina D}

Estudos têm demonstrado que a dificuldade em consumir vitamina D não afeta apenas os vegetarianos, pois é difícil de sintetizar na pele e é muito baixa em alguns alimentos (Baena, 2015). As vitaminas D2 e D3 têm o efeito de suplementar e enriquecer os alimentos. A vitamina D2 (ergocalciferol) é obtida irradiando ergosterol em leveduras com luz ultravioleta e é considerada para uso por vegetarianos, enquanto a vitamina D3 (colecalciferol) é derivada de animais e não é usada em comida vegetariana (Craig \& Mangels, 2009).

Suplementar 5 a $10 \mathrm{mg}$ de vitamina D por dia é seguro e suficiente (Borges et al., 2015). O alimento é fortificado, como algumas bebidas vegetais como soja e arroz (Craig \& Mangels, 2009). Recomendado para homens e mulheres ingerir 5 mg por dia (Castillo \& Ortigosa, 2017), porém a principal e melhor forma de obtenção dessa vitamina é através da exposição solar (Slywitch, 2012).

É citado por Baena (2015), que a ingesta da Vitamina D na população aderente ao estilo de vida vegetariano é baixo, no entanto, é citado pelo autor que as taxas dessa vitamina em ambos os organismos não apresentam diferença.

\subsubsection{Cálcio}

A ingesta do cálcio apresenta uma alta relação com a ingesta da Vitamina D, pois os dois exercem um importante papel no sistema ósseo, contribuindo ainda na manutenção dos sistemas nervoso, muscular e diretamente na coagulação sanguínea (Borges et al., 2015; Pedro, 2010). Na população ovolactovegetarianos e onívoros, a ingesta de cálcio não reduz, no entanto em indivíduos veganos esse componente é menor (Baena, 2015).

Sabe-se que a biodisponibilidade do cálcio sofre variação de acordo com cada componente alimentar, sendo o leite do boi o mais abundante neste componente. Quando comparado com o leite de soja, chegou-se a conclusão de que a soja possui 
apenas $75 \%$ da biodisponibilidade do bovino e, sendo necessário $500 \mathrm{mg}$ de cálcio suplementados para chegar ao leite bovino (Almeida, Buzinaro \& Mazeto, 2006).

É citado por Slywitch (2012), que não há diferença alguma na recomendação diária de ingestão de cálcio, sendo 1000mg/dia, tanto para homens de 19 a 70 anos, como para mulheres de 19 a 50 anos. Sendo assim, há a possibilidade de atingir as doses recomendadas diariamente através da ingesta de alimentos com alta biodisponibilidade de deste nutriente, como por exemplo os brócolis, repolho chinês e couve, além de alimentos enriquecidos com cálcio como bebidas vegetais de soja, amêndoas, arroz e aveia.

\subsubsection{Proteínas}

As proteínas são consideradas macronutrientes que apresentam uma função principalmente estrutural, sendo essencial para o sistema muscular, pois auxilia na reparação celular e na transmissão das sinapses nervosas. Esse componente é encontrado principalmente na carne, no entanto, a soja oferece um valor biológico próximo, podendo sua obtenção se dar de diferentes formas, como no isolado proteico que, em $30 \mathrm{~g}$, possui $26,4 \mathrm{~g}$ de proteína e o tofu que, em $113 \mathrm{~g}$ fornece de $8 \mathrm{~g}$ a 12 g de proteína para o organismo humano (Couceiro, Lenz \& Slywitch, 2008; Crowe, et al. 2016).

Borges et al., (2015), cita que além dos produtos à base de soja, há a possibilidade de encontrar uma variedade de proteína em alimentos de origem vegetal, como através das leguminosas, cereais integrais, quinoa, amaranto. Caracterizando como possível essa obtenção nutreica.

De acordo com o Guia Alimentar de Dietas Vegetarianas para Adultos, para se obter a quantidade recomendada de lisina, pode-se ingerir 4 colheres de sopa de feijão. No caso da ingestão diária proteica, indica-se a quantidade de 10 a $15 \%$ do Valor Calórico Total. É complementado por Slywitch (2012), que a ingesta proteica de indivíduos ovolactovegetarianos e vegetarianos estritos ficam entre 12\% a 13,8\% do Valor Calórico Total, e a de indivíduos onívoros ficou entre 14,8\% e 16,3\% do Valor Calórico Total, sendo assim notável que mesmo com uma ingesta diminuida, os vegetarianos ingerem a quantidade de proteína recomendada como diária.

\subsubsection{Ferro}

O ferro é considerado um nutriente primordial na produção de hemoglobinas, podendo sua ingesta ser de duas formas: através do ferro heme e ferro não heme. $O$ ferro não heme pode ser encontrado nos alimentos vegetais, o qual apresenta maior sensibilidade tanto nos inibidores, quanto nos estimuladores da absorção do ferro (Craig \& Mangels, 2009). O ferro heme, pode ser obtido através da ingesta de alimentos cárneos, o qual possui o anel porfirínico, que tem como função o auxilio na absorção constante do ferro na estrutura gastrointestinal humana (Slywitch, 2012; Andrade et al., 2017).

É citado por Craig \& Mangels, (2009), que a biodisponibilidade de ferro na dieta vegetariana é menor do que na dieta onívora, sendo necessária a ingesta de 5,5 a 11,1 mg de ferro, para que ocorra a absorção de 1 a $2 \mathrm{mg}$ de ferro no organismo, e na dieta vegetariana, seria ideal a ingestão de 8,3 a $16,6 \mathrm{mg}$ de ferro, o mais recomendado seria a ingesta de $16 \mathrm{mg}$ de ferro para homens vegetarianos acima de 19 anos, e $36 \mathrm{mg}$ para mulheres vegetarianas de 19 a 50 anos. Já nas mulheres, a ingestão de ferro apresenta-se abaixo do recomendado, considerando não somente fatores nutricionais, mas também os fatores biológicos (Slywitch, 2012; Adami, Fassina \& Hauschild, 2015),

Nota-se que é incidente a anemia pela diminuição de ferro em vegetarianos, sendo semelhante em onívoros (Craig \& Mangels, 2009), no entanto, a ingesta de ferro em vegetarianos apresenta-se em quantidade maior do que a de onívoros, levando em conta, a maior ingestão de alimentos ricos em vitamina $\mathrm{C}$ feita por esses indivíduos (Couceiro, Lenz \& Slywitch, 2008; Chauveau, et al. 2016). 


\subsubsection{Vitamina B12 (Cobalamina)}

A vitamina B12 é considerado um dos nutrientes que requerem maior atenção dos vegetarianos e veganos, pois é encontrada principalmente em alimentos de origem animal, levando assim a deficiência nutricional desse componente, aneminas e até deficiências neurológicas (Marques et al., 2016; Borges et al., 2015).

É citado por Craig e Mangels (2009), que o ácido fólico, por ser presente em grandes quantidades na dieta vegetariana, pode ocultar sintomas da deficiência de vitamina B12, e aparecer somente, com o passar do tempo através de sintomas neurológicos mais graves.

De acordo com Slywitch (2012), pode-se obter uma maior quantidade de vitamina B12 em alimentos de origem animal, sendo o queijo proveniente do porco o alimento com maior teor de B12, além disso, é enfatizado que a ingestão de algas não ajuda na recomendação diária desta vitamina, pois são consideradas metabolicamente inativas em mamíferos.

\subsection{A importância do profissional de nutrição no acompanhamento desta filosofia alimentar}

Segundo o Ministério da Saúde (há uma ligação direta entre nutrição, e a saúde, por isso é necessário ter uma alimentação balanceada tendo todos os nutrientes que ajudam no funcionamento do corpo. Nessas dietas mais restritivas conquistar esse equilíbrio acaba sendo uma tarefa mais difícil, entretanto com a ajuda de um profissional da nutrição o objetivo será traçado com maior facilidade. "Uma das maiores preocupações referentes à alimentação vegetariana é a adequação nutricional. A exclusão de origem animal que pode ou não predispor carências nutricionais.” (Sezini, 2020, P.2).

$\mathrm{O}$ atendimento nutricional realizado em uma pessoa vegetariana tem basicamente todos os aspectos de uma consulta com pacientes com dietas normais, buscado sempre a melhor maneira de organizar a alimentação. A partir do primeiro contato e das trocas de experiências, deve ser feito todos os procedimentos técnicos para avaliação do estado nutricional do paciente, envolvendo anamnese, recordatório de $24 \mathrm{~h}$, analise de exames de sangue, e antropometria para assim traçar um esquema de acompanhamento nutricional (Gonçalves, 2017; Biarge, 2018).

A proteína encontrada nos animais normalmente dispõe de todos os aminoácidos essenciais sendo sua maior diferença em relação a proteína encontrada nos vegetais, o que pode causar certo receio ao paciente. Como não possuem todos os aminoácidos essenciais que se precisa é importante misturar dois ou mais fontes da proteína vegetal, como o arroz e o feijão. O aminoácido que falta em um desses alimentos tem no outro (Pandovani, 2019; Braga, et a., 2017).

O nutricionista saberá fazer essas combinações, pois possuem informações sobre os aminoácidos essenciais e poderá mudar elas diversas vezes pra que, a alimentação não se torne algo corriqueiro. Para os ovo-lacto-vegetarianos as determinadas recomendações das proteínas já são atingidas com os ovos, leites e derivados (Sezini,2020; Burns-whitmore, et al. 2016). Para identificar uma carência de proteína no sangue deve ser feito por um profissional a dosagem da albumina sanguínea, a transferrina ou também a contagem de linfócitos, que demonstram em termos de proteína o estado nutricional.

Assim como a proteína vários nutrientes possuem fonte principal em alimentos provenientes da carne animal. Sabe-se que ferro heme é melhor absolvido. O outro ferro (não heme) estar nos vegetais e a absorção dele é mais lenta por isso alguns vegetarianos pode vir a ter anemia ferropriva, pois não consome a quantidade necessária por dia. O nutricionista saberá aumentar a biodisponibilidade desse mineral através da junção da vitamina $\mathrm{C}$ que auxilia na absorção desse nutriente (Sezini, 2020; Baroni, et al. 2018). Se através dos exames for identificada a deficiência do mineral ferro, não poderá ser corrigida apenas pela alimentação e sim pelo ferro medicamentoso, seja em vegetarianos ou não, por tempo prolongado, em doses que normalmente não se alcançam somente com as refeições (Slywitch, [20--], p.11).

\subsubsection{Nutricionista e a suplementação para os vegetarianos}

A filosofia vegetariana por si só não causa malefícios a saúde em nenhum estágio da vida, incluindo, portanto, as 
crianças, adultos e idosos, gestantes, lactantes e também atletas. Em alguns casos a suplementação determinada pelo nutricionista é necessária para garantir a saúde. O que de certa forma não define se a dieta é menos saudável (Minari, 2019; Armelagos, 2016).

Com a ajuda de um nutricionista pode-se suprir quase integralmente os nutrientes apenas na base da alimentação e sem nenhuma interferência medicamentosa, contudo a vitamina B12 é a única que deve ser suplementada na alimentação dos vegetarianos estritos (Minari, 2019, P.03). "As únicas fontes confiáveis de B12 são as carnes, ovos, leites, queijos, alimentos enriquecidos e suplementos." (Sezini,2020, P.05). A suplementação é capaz de ser utilizada diariamente contendo no mínimo 5 mcg da vitamina B12. O profissional responsável ao analisar o hemograma pode não identificar a carência (anemia megaloblástica). Deve ser solicitada dosagem de vitaminas B12 sérica, também dosar a homocisteina e se necessário o ácidometilmalônico. A suplementação normalmente se dá por via oral, podendo ser injetável apenas se o paciente apresentar anemia perniciosa (Slywitch, 2017; Appleby, Key, 2016).

O valor da recomendação do ômega-3 para os vegetarianos é duas vezes maior do que para os onívoros. O Ômega-3 dos vegetais precisa ser convertido a EPA e DHA, o que diferencia do ômega provenientes dos peixes (Sezini, 2020; Andres, et al. 2016). A suplementação desse nutriente pode de feita através do óleo de alga que é considerado uma fonte rica de DHA, além de ser vegana (Minari, 2019; Ali, et al. 2017).

Dado os fatos a orientação nutricional resultante de um nutricionista surge para agregar e facilitar a alimentação denominada vegetariana e vegana. Prevenindo possíveis carências por escassez de informação e abrindo possibilidade para novos alimentos e combinações. Incluir o nutricionista como parte essencial desse estilo de vida ameniza a possível impressão negativa do vegetarianismo (Abonizio, 2016; AgnolI, et al. 2017).

\section{Conclusão}

Espera na realização desse projeto orientar os praticantes do vegetarianismo sobre a importância do nutricionista ao iniciar essa espécie de alimentação, pontuando os riscos da falta de informação que pode causar inúmeras desordens alimentares, como as deficiências nutricionais que podem ser supridas tanto pela dieta, adequando os nutrientes e respeitando as recomendações quanto, e se preciso, fazer o uso da suplementação. Dessa forma é importante pontuar que nessa orientação mostra-se que essa filosofia não é prejudicial para a saúde e pode ser benéfica nos estágios da vida.

\section{Referências}

Abbud, K., et al. (2018). Nutrientes e suas funções: equilíbrio é fundamental quando se trata de alimentação saudável. Alimentação em foco. https://alimentacaoemfoco.org.br/nutrientes-e-suas-funcoes/.

Adami, F. S., Fassina, P., \& Hauschild, L. (2015). Estado nutricional e qualidade da dieta em indivíduos vegetarianos estritos e não-estritos. Revista Uningá Review, 23(2), 18-24.

Almeida, R. N. A., Buzinaro, E. F., \& Mazeto, G. M. F. S. (2006). Biodisponibilidade do Cálcio Dietético. Arq Bras Endocrinol Metab, 50(5);

Andrade, J. S. (2018). Dieta vegetariana: riscos e benefícios à saúde. Monografia, Universidade Federal de Pernambuco, Vitória de Santo Antão.

Abonizio, J. (2016). Conflitos à mesa: vegetarianos, consumo e identidade. Revista Brasileira de Ciências Sociais, 31(90), $115-137$.

AgnolI, C., et al. (2017). Position paperonvegetarian diets fromtheworkinggroupofthe Italian Society ofHumanNutrition. Nutrition, Metabolismand Cardiovascular Diseases, 27(12), 1037-1052.

Ali, A. B. G., Andrade, B. C. P., Oliveira, M. M. S., et al. (2016). Introdução alimentar em crianças veganas - Revisão de literatura. Ver Uningá Review. 20(3)82-87.

Andres, E. et al. (2016). Vitamin B12 (cobalamin) deficiency in elderlypatients. CMAJ, 171(3), 251-9.

Appleby, P.; \& Key, T. (2016). The long-term health of vegetarians and vegans. Proceedings of The Nutrition Society, 75(3), $287-293$. 
Armelagos, G. (2016). Brain evolution, the determinates of food choice, and the omnivore's dilemma. Critical Reviews in Food Science and Nutrition, 54(10), $1330-1341$

Azevedo, E. (2013). Vegetarianismo. Demetra: alimentação, nutrição e saúde. Demetra: food, nutrition \& health. 8(Supl.1), 27588. https://doi.org/10.12957/demetra.2013.6609. https://www.e-publicacoes.uerj.br/index.php/demetra/article/view/6609/5757.

Andrade, R. M., Brauna, C., Cavagnari, M. A. V., Noronha, B. T., Oliveira, C., \& Silva, T. (2017). Avaliação qualitativa de nutrientes na alimentação vegetariana. Revista UNINGÁ Review, 29(1), 222-226,

Baena, R. C. (2015). Dieta vegetariana: desafios e benefícios. Diagnóstico \& Tratamento, 20(2), 56-64 2014. http://www.apm.org.br/imagens/Pdfs/revista140.pdf

Barranha, S. (2017). Alimentação Vegetariana em Idade Pediátrica: riscos, benefícios e recomendações (Dissertação/Artigo de revisão bibliográfica Mestrado Integrado em Medicina). Instituto de Ciências Biomédicas Abel Salazar, https://repositorio-aberto.up.pt/bitstream/10216/109780/2/238265.pdf

Baroni L., Goggi S., Battaglino R., et al., Vegannutrition for mothersandchildren: practical tools for healthcare providers. Nutrients.

Burns-whitmore, B., et al. (2016). Effects of supplementing n-3 fatty aciden riche deggs nad walnutson cardiovascular disease risk markers in healthy freeliving lacto-ovo-vegetarians: a randomized, crossover, free-living intervention study. Nutr J, 13,29.

Biarge M. M. Ninõs vegetarianos, ¿ninõssanos? Curso de ActualizaciónPediatría. Madrid: LúaEdiciones 3.0. 2018;223-234.

Braga J. A. P., et al. editores. (2017). Guia de Nutrição Clínica na Infância e na Adolescência. Manole, 219-41.

Beig, B. B. (2009). A prática vegetariana e os seus argumentos legitimadores: viés religioso. Revista Nures. 11. < http://www.pucsp.br/nures/Nures11/artigo_beatriz.pdf>.

Baiochi, A. W. A., \& Cardoso, C. K. S. (2020). Efeito padrão alimentar vegano ou vegetariano em fatores cardiometabolicos: uma revisão de literatura científica. 19 f. TCC (Graduação) - Curso de Nutrição, Puc Goiás, Goiás, https://repositorio.pucgoias.edu.br/jspui/handle/123456789/694.

Borges, C., Graça, P., Pinho, J. P., Santos, A., Santos, C. T., Silva, S. G. C. (2015). Linhas de orientação para uma alimentação vegetariana saudável. Lisboa: Programa Nacional para Promoção da Alimentação Saudável.

Castillo, A. T., \& Ortigosa, R. S. (2017). A propósito de un caso: Dietas Vegetarianas. Atualización en Medicina de Familia, 13(6), $320-326$.

Chauveau, P. et al. (2016). Vegetarianism: advantages and drawbacks in patients with chronic kidney diseases. Journal of Renal Nutrition, 23(6), 399-405.

Crowe, F. et al. (2016). Plasma concentrations of 25-hydroxyvitamin D in meat eaters, fish eaters, vegetarians and vegans: results from the EPIC-Oxford study. Public Health Nutrition, Cambridge, 14(2), 340-346.

Cavalheiro, C. A., Verdu, F. C., \& Amarante, J. M. (2018). Difusão do vegetarianismo e veganismo no brasil a partir de uma perspectiva de transnacionalização. 6(1), $51-67$.

Couceiro, P., Slywitch, E., Lenz, F. (2008). Padrão alimentar da dieta vegetariana. Einstein http://apps.einstein.br/revista/arquivos/PDF/518v6n3aRW518portp365-73.pdf.

Cozzolino, S. M. F. (2016). Biodisponibilidade de Nutrientes. (5a ed.), Manole.

Craig, W. J., \& Mangels, A. R. (2009). Position of the American Dietetic Association: Vegetarian Diets. Journal of the American Dietetic Ssociation, 109(7), 1266-1282

Dagostin, C. T., Rigo, F. K., \& Damázio, L. S. (2019). Associação entre alimentação vegetariana e a prevenção do câncer colorretal: uma revisão de literatura. Revista Contexto \& Amp; Saúde, 19(37), 44-51. https://doi.org/10.21527/2176-7114.2019.37.44-51.

Damante, A. L., M, F. (2017). O papel do nutricionista no aconselhamento dietético de atletas vegetarianos. 7(5), 95-106

Elmadfa, I., \& Singer, I. (2016). Vitamin B-12 and homocysteine status among vegetarians: a global perspective. The American Journal of Clinical Nutrition, 89(5), 1693S-1698S.

Ferrigno, M. (2016). Veganismo e libertação animal: um estudo etnográfico. Dissertação de Mestrado. Universidade Estadual de Campinas.

Fuller, J. (2016). The cultural creation of affect amongst vegan consumers: Aneth no graphic analysis of online consumption communities. Departmentof Service Management and Service Studies, LundUniversity.

Fabres, Rosa. (2016). Dieta Vegetariana: razões e impactos na saúde, [S. L.], 1-38.

Franco, E. S., \& Rego, R. A. (2005). Marketing estratégico para subculturas: um es tudo sobre hospitalidade e gastronomia vegetariana em restaurantes da cidade de São Paulo. Turismo - Visão e Ação - 7(3), 469 - 482

Freitas, L., \& Pamela, P. (2018). "A construção social da alimentação: a vegetariana e o veganismo na perspectiva da psicologia histórico-cultural”.

Fofonka, L. W., \& Machado, S. (2017). Carne ou vegetais? Os benéficios do vegetarianismo para a saúde e para o meio ambiente, [s. 1.]. http://www.revistaea.org/artigo.php?idartigo $=2782$.

Gomes, C. F., Guerra, F. M. R. M., Junior, J. M., Simão, A. N. C., \& Vaz, D. S. S. (2014). A importância do ômega 3 para a saúde humana: um estudo de revisão. Revista Uningá Review, 20(2), 48-54. 
Gonzaga, F. (2006). Cozinha Vegetariana: A alternativa saudável, Editora Impala, 1, 6 - 25.

Guimarães, G. (2010). Sobre nutricionista e a resistência ao vegetarianismo. Nutriveg [página na internet]. Rio de Janeiro. http://www.nutriveg.com.br/sobrenutricionistas-e-a-resistecircncia-ao-vegetarianismo.html.

Hauschild, Adami, \& Fassina. (2015). Estado nutricional e qualidade da dieta em Indivíduos vegetarianos estritos e não - estritos. Revista UNINGÁ Review, 23(2), 18-24.

Hyde, N. et al. (2017). Maternal nutrition during pregnancy: intake of nutrients important for bone health. Maternal and Child Health Journal, 21(4), 845-851.

Krey, I., Pereira, D., Desiree, D., Mendes, M., Mendes, M., Galvão, B., Renata Furla, D. S. C. (2017). Atualidades sobre dieta vegetariana.

Leão, A. L. M; Santos, L. C. Consumo de micronutrientes e excesso de peso; existe relação? Rev. bras. epidemiol. São Paulo. 15(1), 85-95

Lopes, M. S. et al. Iodo e tireoide: o que o clínico deve saber. Revista Científica da Ordem dos Médicos, 3(25), $174-178$.

Mata, G. K. S., et al. (2020). O impacto da dieta vegana na saúde: revisão sistemática. 14 f. TCC (Graduação) - Curso de Nutrição, Centro Universitário Tiradentes - Unit, Alagoas. https://openrit.grupotiradentes.com/xmlui/handle/set/3905.

Magluf, Dieta Vegetariana: Razões E Impactos Na Saúde. dieta-vegetariana-razoes-e-impactos-na-saude-nicole-m-m-r-febres.pdf

Marques, A. (2015). Rota gastronômica vegetariana /vegan ibérica. 33f. Trabalho final (Curso intensivo universitário sobre patrimônios alimentares, cocinas patrimonales y turismo gastronômico. Universidade de Barcelona, Barcelona.

Marques, Núria Araújo. (2020). Vegetarianismo e veganismo na educação: investigação de experiências no contexto escolar brasileiro = Vegetarianism and veganism in education: investigation of experiences in the Brazilian school context. 1 recurso online, 147. Dissertação (mestrado) - Universidade Estadual de Campinas, Faculdade de Educação, Campinas, SP. http://www.repositorio.unicamp.br/handle/REPOSIP/361176.

Marques, P. F., Martins, J. A., Rodrigues, D., Silva, M. A., Siqueira, E. P. (2016). Avaliação da oferta nutricional de dietas vegetarianas do tipo vegana. Revista Intellectus, 1(33).

Martins, M. (2011). Nutrição vegetariana: avanços e perspectivas. Life Style, 1(2), 8-10. https://unasp.emnuvens.com.br/LifestyleJournal/article/view/123.

Medeiros, Maria Lima. (2017). Quais as influências para as pessoas adotarem estilo de vida vegano, [S. L], 1- 36.

Nadal, J., \& Mezzomo, T. R. (2016). Efeit o dos nutrientes e substâncias alimentares na função tireoidiana e no hipotireoidismo. De metra: Food, Nutrition \& Health / Alimentação, Nutrição \& Saúde, 11(2), 427-443.

Nascimento, I. S., \& S Awyer, D. (2007). Vegetarianos do Brasil: Consumo x Produção de carne. Centro de Desenvolvimento Sustentável. Universidade de Brasília. http://www.unbcds.pro.br/conteudo_arquivo/090708089C57.pdf>.

Navolar; T., \& Azevedo. (2012). Contribuições para a construção da nutrição complementar integrada. 16(41), 515 - 27.

Negri, S. T., De Alves, L. G. (2018). Abordagem sobre o vegetarianismo na formação do profissional nutricionista. https://www.guiavegano.co $\mathrm{m}$.br/vegan/acervo-vegano/downloads/artigos/155-artigo-abordagem-sobre-vegetarianismo-na-formac-a-o-do-profissional-nutricionista-so-nia-teresinha-denegri.

Orlich, M. J., \& Fraser, G. E. (2014). Vegetarian diets in the Adventist Health Study 2: a review of initial published findings. The American Journal of Clinical Nutrition, [s. 1.], 100, 353S-358S.

Padovani, Rosália. (2019). Proteína animal ou vegetal.

Perovano, D. G. (2014). Manual de Metodologia Científica. Editora Juruá.

Pimentel D., Tomada I., \& Rêgo C. (2018). Alimentação vegana nos primeiros anos de vida: considerações e orientações [trabalho de conclusão de curso]. Porto: Universidade Católica Portuguesa;

Pinho, et al. (2016). Guia Alimentar Mediterrânico: Relatório justificativo do seu desenvolvimento. Programa Nacional para a Promoção da Alimentação Saudável. Direção-Geral da Saúde.

Perrota, Ana Paula. (2017). Vegetarianismo ético e posições carnívoras: questões além do sabor e dos nutrientes. Estudos Sociedade e Agricultura, 25(2)., 327352. Universidade Federal Rural do Rio de Janeiro, Brasil.

Pinheiro, I., et al. (2018). Atualidades sobre dieta vegetariana. Nutrição Brasil, 6(16), 406-413, https://portalatlanticaeditora.com.br/i ndex.php/nutricaobrasil/article/view/744.

Queiroz, C. A., Soliguetti, D. F. G., \& Moretti, S. L. A. (2018). As principais dificuldades para vegetarianos se tornarem veganos: um estudo com o consumidor brasileiro. 20 f. TCC (Graduação) - Curso de Marketing, Não Informa, São Paulo. https://www.e-publicacoes.uerj.br/index.php/de metra/article/download/33210/26420.

Ramos, A C. (2019). Biodisponibilidade de nutrientes na alimentação vegetariana, [S. L.], 1-17, https://repositorio.uniceub.br/jspui/bitstrea m/prefix/13481/1/21603897.pdf

Révillion, J. P. P., et al. (2020). O mercado de alimentos vegetarianos e veganos: características e perspectivas. Caderno de Ciência e Tecnologia, Brasília, 37(1), 1-10: https://seer.sct.embrapa.br/index.php/cct/article/view/26603.

Rodrigues, C. (2005). Introdução ao vegetarianismo. (2a ed.), Edição e distribuição: galaxia-alfa.com. 
Research, Society and Development, v. 10, n. 13, e148101321112, 2021

(CC BY 4.0) | ISSN 2525-3409 | DOI: http://dx.doi.org/10.33448/rsd-v10i13.21112

Sezini, M. (2020). Vegetarianismo. Suplementação nutricional. Fitoterapia. Noções de farmacologia. www.estrategiaconcursos.com.br.

Slywitch, E. (2012). Guia alimentar de dietas vegetarianas para adultos. https://www.svb.org.br/livros/guia-alimentar.pdf (livro). SVB.

Spencer, E. A., et al., (2003). Diet and body mass index in 38000 EPIC-Oxford meat-eaters, fish-eaters, vegetarians and vegans. Int J Obes Relat Metab Disord, 27(6), 728-34.

Tong, T. Y. N, et al. (2020). Dietas vegetarianas e veganas e riscos de fraturas totais e específicas do local: resultados do estudo prospectivo EPIC-Oxford. 18(353). 